\title{
PERCEPTIONS OF THE ACADEMICS FROM FACULTY OF SCIENCE, PALACKÝ UNIVERSITY OLOMOUC TOWARDS SELECTED ASPECTS RELATED TO THE COURSE OF THE COVID-19 PANDEMIC IN THE CZECH REPUBLIC AS AN EXAMPLE OF A SUCCOUR FOR THE STRATEGY AND POLICY MAKERS
}

Pavel RUŠAR $\varangle$, Faculty of Science, Palacký University Olomouc, 17. listopadu 1192, Olomouc, pavel.rusar01@upol.cz

Radim KAPAVÍK, Alma mater - Faculty of Information Technology, Brno University of Technology, Antonínská 1548, Brno, kapavikr@gmail.com

Keywords: COVID-19 pandemic in the Czech Republic, Faculty of Science UPOL, perceptions of the academics towards the COVID-19 pandemic, survey as a tool in public health

\begin{abstract}
:
Background: The impact of the COVID-19 illness all around the world is enormous and as such is a subject of study for not only medicine but also geography, economics, society, psychology and media. Information, data and materials which are the basis for decisions made by policy and strategy makers are acquired from a vast and chaotic mixture of sources. Those are produced based on opinions of experts from diverse fields, results of statistical or clinical studies, official or covert economic interests, political opinions or media pressure and many more inputs. This study presents a concrete example of a possible instrument for correction for the influence of the potentially misleading view in media or society.
\end{abstract}

Methods: Using a questionnaire, this study heuristically quantifies the perceptions of a selected group of Faculty of Science, Palacký University Olomouc employees towards the development of the COVID-19 pandemic in the Czech Republic. We presume that overall they possess more experience with critical analysis and recognizing the relevance of the data than the general population.

Results: The respondents assume that based on the data they believe to be relevant COVID19 is less than or comparably dangerous as seasonal flu (34.1\%) or several times or by an order of magnitude more dangerous $(54,1 \%)$; implementing wearing face covering inside is in total for the population rather beneficial $(83,8 \%)$, or disadvantageous or without any significance $(7,5 \%)$, the daily count of infected is relevant $(49,7 \%)$, or irrelevant $(42,2 \%)$; the exposure rate in the population is relevant $(55,2 \%)$, or irrelevant $(34,0 \%)$; the percentage of the Czech population that has already been exposed to the virus is $5 \%$ or less $(46,5 \%)$, or $6 \%$ or more $(29,2 \%)$. The respondents view the government measures as relevant and sufficient or insufficient $(58,9 \%)$, or relevant but excessive $(20,0 \%)$, or irrelevant and excessive or absurd $(9,2 \%)$. A significant amount of further data has been derived from the results presented in this study.

Conclusion: Certain results of the perceptions quantification reflect the predominant media discourse (such as the question of the benefit of wearing face coverings inside), other seem to differ or contradict the discourse (such as the comparison with seasonal flu or the preference for the exposure data over the daily infected count). As a conclusion, it can be ascertained that when correctly implemented a verified survey of a predefined closed group of respondents can be an applicable instrument for a potential correction for the prevailing public and media paradigm. 


\section{INTRODUCTION}

COVID-19 was first observed at the end of 2019 in Wuhan, China. It is caused by a previously unknown type of RNA coronavirus, that is now referred to by the academia as SARS-COV-2. ${ }^{1}$ The disease spread rapidly into various regions of China and further to other continents. On 1 March 2020 its presence in the Czech Republic was confirmed and on 11 March WHO described the spread of this disease as pandemic. ${ }^{2}$ It is accepted in the medical community that the virus spreads when people are in close contact (mainly by droplet infection). Among the main symptoms of the disease are cough, shortness of breath, fever, muscle aches and fatigue. The degree of seriousness differs greatly, from almost imperceptible to severe pneumonia that can pose a threat to patients' lives. ${ }^{3}$

Due to the massive spread of COVID-19 and the number of infected, the medical community as well as the scientific community across the world focused on understanding both the virus and the disease. The research is aimed at the course of the illness, the transmission, risk factors for complications, long-term effects, comorbidities, efficacy of protective equipment and many more. ${ }^{4}$

Political representatives and managers in the health services were and still are faced with a lack of data when making decisions, or with the insufficient credibility, relevance, or stringency of the data. A number of epidemiologic measures around the world were taken without adequate data, as those were unavailable or inaccessible, or strategy and policy makers did not utilize them for one reason or another. The pandemic is strongly affecting businesses and the economy of the majority of countries, as well as the media. The relations between the media and the measures taken against the spread of the disease were the subject of numerous studies in various fields. Social scientists embarked to examine various hypotheses on the connections between the transmission and geographic, social, economic, demographic, and other determinants. ${ }^{5}$ Sociologists and other scientists around the world began to study the mood and attitudes of the population. ${ }^{6}$ This study would like to expand on these works and add a new angle of a survey of perceptions current among the academics.

The main intent of the study is to utilize a concrete example and to outline whether or in what way could quantification of the position of a predetermined segment of the population be beneficial or relevant for the policy and strategy makers or for the management in health care. This concrete example consists of a heuristic attempt to quantify the perception of a selected sample of employees from the Faculty of Science, Palacký University Olomouc (FoS UPOL) to certain aspects related to the evolution of the COVID-

\footnotetext{
${ }^{1}$ International Committee on Taxonomy of Virus. Naming the 2019 Coronavirus (2020) https://talk.ictvonline.org/, April 2020.

${ }^{2}$ Ministry of Health of the Czech Republic, https://koronavirus.mzcr.cz/v-ceske-republice-jsou-prvni-tripotvrzene-pripady-nakazy-koronavirem/, September 2019; Cucinotta D, Vanelli M. WHO Declares COVID-19 a Pandemic. Acta Bio Med [Internet]. 2020Mar.19 [cited 2020Sep.27];91(1):157-60. Available from: https://www.mattioli1885journals.com/index.php/actabiomedica/article/view/9397

${ }^{3} \mathrm{Ge}, \mathrm{H}$., Wang, X., Yuan, X. et al. The epidemiology and clinical information about COVID-19. Eur J Clin Microbiol Infect Dis 39, 1011-1019 (2020). https://doi.org/10.1007/s10096-020-03874-z

${ }^{4}$ Wide variety of published results can be found for example in the PubMed database;

https://pubmed.ncbi.nlm.nih.gov/, September 2020.

${ }^{5}$ Wide variety of published results can be found for example in the PubMed database; https://pubmed.ncbi.nlm.nih.gov/, September 2020.

${ }^{6}$ An example of such research is the National Pandemic Alarm project, https://www.nationalpandemicalarm.eu/main-cs.html, September 2020.
} 
19 pandemic in the Czech Republic. This is the main research aim of the study. The motivation behind this survey among the employees of the FoS UPOL is an attempt to ascertain opinions of a portion of the population that does not have a medical education as such, but simultaneously due to their occupation and education in natural sciences possesses considerably more experience with discerning the quality and relevancy of data than the general population.

The claim that academic personnel of the FoS UPOL are better able to search for good quality data, to better distinguish between the sources according to their reliability and to assess the relevancy of data than the general population is not proven in this study, we treat it as an axiom. The result of this survey is not any claim about which opinions are more real or true, but a quantification of convictions of FoS UPOL employees as respondents who as a part of their work process evaluate data and information. In this study we do not compare our results with any survey of other sub-parts of the population, although we consider obtaining such data for a comparison.

Apart from the quantification of opinions of FoS UPOL employees through their responses, the study has also secondary research questions that are connected to it. Do the respondents conclude that they possess a sufficient amount of reliable data related to predetermined assertions regarding the events connected to the pandemic? Will the respondents emphasize the importance or superiority of data published in peer-reviewed and impacted journals? How will the results differ, when we compare answers by employees who work in fields that are by definition closer to the medical and biological aspect of the pandemic (herein after referred to as more related fields) with answers by employees from fields that are not that closely related (herein after referred to as less related fields)? Will statistically interesting data clusters emerge? And if so, what would it suggest?

What will be the ratio between natural scientists, who rather do not agree with implemented blanket measures and presume that the disease does not pose such a serious health threat and that the rate of the exposure in the population is high with natural scientists who on the other hand presume that the measures are correct, the disease poses a very significant threat and that the exposure in the population is not very high?

From the aims and research questions of this study arises what the research does not explore. The study does not aim to contribute to the discussion of the perilousness of contracting the SARS-CoV-2 virus, the COVID-19 illness, the exposure rate of the Czech population or the comparison with the seasonal flu. The research also does not reflect the measures adopted by the Czech government, their assessment, nor the discussion about their efficaciousness. The results of this study cannot be utilized as an argument in these or related areas. In the conclusion it will be discussed under which conditions and using what potential data could the result be utilized by the policy and strategy makers or the management in the healthcare sector. 


\section{METHODS}

To establish the attitudes FoS UPOL employees hold, we utilised an online survey. ${ }^{7}$ The survey was divided into three parts. In the introductory part the respondents were to choose their academic degree, the field of their study and their specialization. The next part asked questions (see the Results below) about the evolution of the COVID-19 pandemic in the Czech Republic and the respondents were to choose the statements that best corresponded to the data they consider to be reliable or that they are not familiar with such data. The third part of the survey asked the respondents their subjective opinion of the epidemiologic measures in the Czech Republic.

In addition to the previously introduced aim of the survey, i.e. to quantify the position of the employees towards selected aspects of the course of the pandemic as shown by their responses, the survey had also secondary research questions. The survey in its second part was to track the ratio of the "I do not know - I do not have relevant data" option to all the other responses. The survey tracked the statistical change detection of the answers correlated to respondents' fields of study. It also studied the ratio of respondents who utilized a certain discourse by implementing predefined paradigms. The study also implemented algorithmic clustering to observe the occurrence of groups of respondents who answered similarly. Both methods are described in respective parts of the Results chapter.

The study also tracked any other qualitative perceptions that could be quantified in some aspect by the use of the comment section in the questionnaire.

The group the survey was sent to is defined as the employees of the Faculty of Science, Palacký University Olomouc. Further narrowing/extending the selection was based on following conditions:

- Technical and administrative staff were not included unless they were academic or pedagogical personnel

- Employees of the Department of Foreign Languages were not included

- Email addresses were collected from the lists publicly available on webpages of the faculty or respective institutes. If the email addresses were not published on these or the email addresses were invalid, they were not included in this study.

- Postgraduate students were not included when they were identified as such on the webpages of their departments, institutes or other workplaces, or when they were not mentioned at all.

- Emeriti were included when their names were published on webpages of the institute where they used to work

- External personnel of the FoS UPOL were not included

- Employees from the CRH and RCPTM research centres were not included unless their names were published on the webpages of departments or institutes, i.e. unless they work there as well

Included in the study were 497 individuals, that is to say they were sent an email informing them about the possibility of participating in the survey. To prevent any manipulation or

\footnotetext{
7 During the research was utilized the free-of-charge software Google Forms, LibreOffice Calc was utilized for the data analysis.
} 
mishandling of the survey (mainly trolling), each respondent was sent a unique code that had to be entered for the result to be valid. The source file with these codes and the list of email addresses were permanently deleted, the participation in the study was therefore anonymous.

Taking into account the manner in which the data were gathered, it cannot be excluded that among the contacted were respondents who did not meet our criteria or vice versa some potential respondents might have been omitted. It would have been only several individuals and their potential answers would not be statistically significant for the study. The advantages of the selected methodology, that is preventing interference from undesirable debasement (trolling), unequivocally preponderate the above-mentioned potential risk.

Electing to send out unique codes to the respondents was an integral part of the research method, where examined panel of respondents is strictly defined and limited, and each member can contribute into the complete result by exactly one vote. 


\section{RESULTS}

The questionnaire was filled in by 188 respondents, 2 did not produce a valid personal code, 1 was eliminated on the basis of one of their response that indicated that they should not have been included in the target group. Admitted into the study were 185 valid answers (respondents). This corresponds to approximately $37 \%$ of the total number of recipients. The absolute majority, namely $167(90.3 \%)$, filled in the questionnaire the day it was sent out (24 September) or the next day ( 25 September). The rest provided their answers within five days.

a) The results of the first part of the questionnaire are indicated in Pic 1.

b) The results of the first part of the questionnaire are indicated in Pic 2.

c) The results of the first part of the questionnaire are indicated in Pic 3.

d) 45 respondents (24\%) used the opportunity to submit a commentary with the questionnaire. Their contributions referred to economic, social, and psychological impacts of the COVID-19 pandemic or the government measures implemented in a reaction to it, to the topic of vaccination, immunization, the threat of overloading the health care system as a result of the pandemic and limiting other areas of the health care, comparisons with influenza, face coverings and their efficacy in preventing the spread of the virus in the population on one hand and their health risks on the other.

Frequently respondents expressed their opinions (radical opinions included) on the current political events, the government of the Czech Republic and criticized their decisions. Commonly critiqued were also the media, i.e. one-sided news coverage, preference for extreme views, exaggeration of the threat, etc. Some respondents commented on data sources, e.g. insufficient sharing of data by the Czech government, general absence of relevant data. Some respondents included links to data they deemed credible. Some respondents also voiced an opinion that it is important to rely on studies published in peer-reviewed journals.

Other respondents voiced their interest in the survey and wished to know to what end it will be used. Some were sceptical and expressed their view that the questions are misleading, formulated with bias or that the questionnaire is vague. Some were of the opinion that the survey is irrelevant, because it does not target the specialists in the relevant field. Other respondents appraised the questionnaire favourably and some were interested in the resulting data.

One comment centred on adverse consequences of excessive usage of disinfectant on the human microbiome. Unlike the other opinions voiced in the comments, this view is not found frequently in the media coverage.

The distribution of comments according to one of the possible features is displayed in Tab 1. 
Tab 1 - Distribution of comments based on predetermined classification

\begin{tabular}{|l|l|}
\hline $\begin{array}{l}\text { THE RESPONDENT COMMENTED } \\
\text { PREDOMINANTLY ON: }\end{array}$ & $\begin{array}{l}\text { Number of comments } \\
\text { (as a per cent of the } \\
\text { total) }\end{array}$ \\
\hline Political situation in the country, government measures & $11(5.9 \%)$ \\
\hline Questionnaire, its criticisms, expectations of it, etc & $11(5.9 \%)$ \\
\hline Health and health care & $10(5.4 \%)$ \\
\hline Other comments (irrelevant, humorous, vulgar, etc) & $7(3.8 \%)$ \\
\hline Data, their relevancy, availability, accessibility, etc & $6(3.2 \%)$ \\
\hline
\end{tabular}

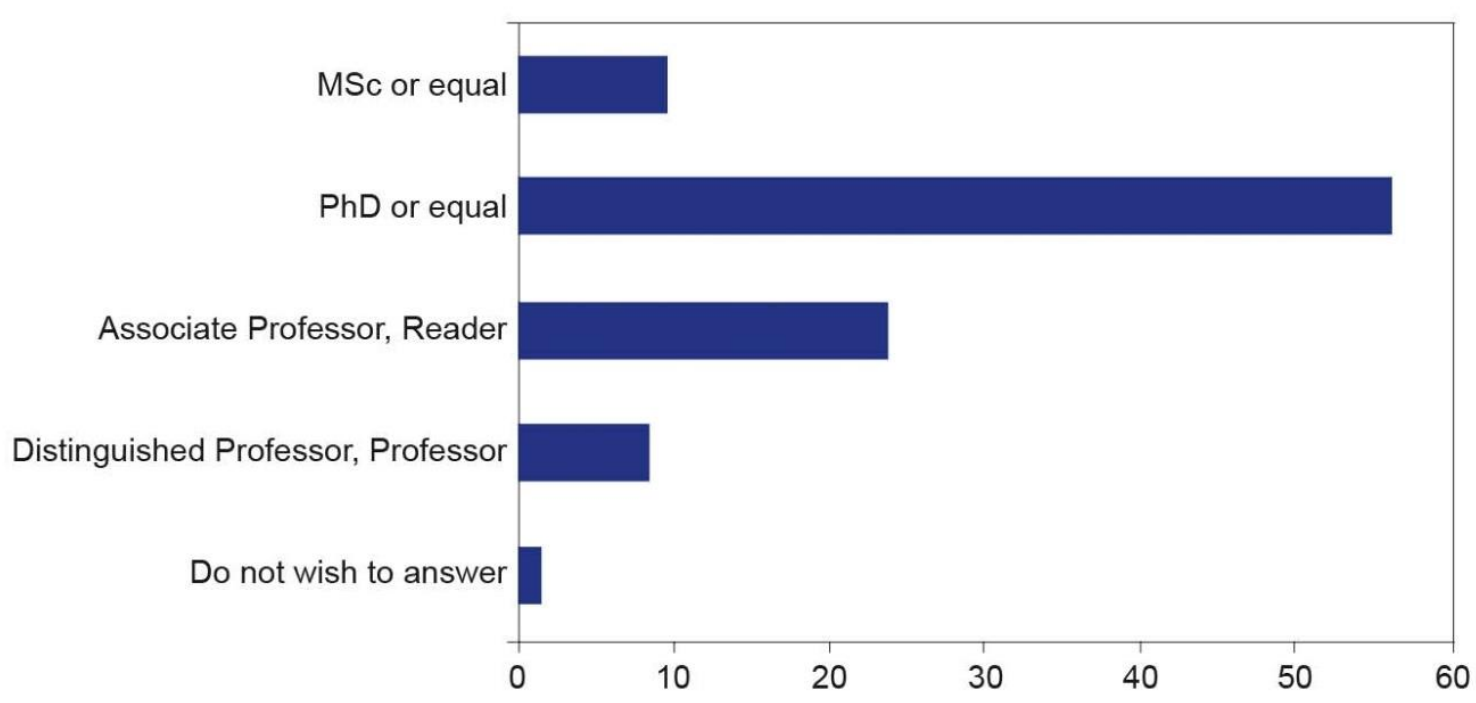

Pic 1 - The answers of the respondents about their highest academic degree achieved (\%) 
1) COVID-19 when compared with the seasonal flu is for the population of the Czech Republic:

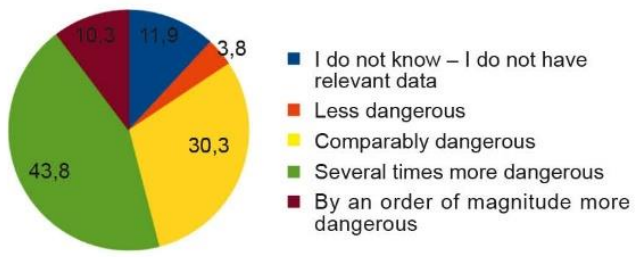

3) Daily updates on the number of detected COVID-19 positive cases are:

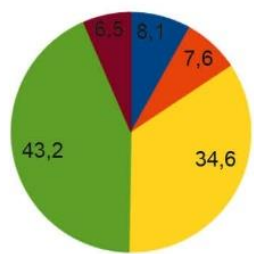

- I do not know - I do not have relevant data

- Completely irrelevant

Rather irrelevant

- Rather relevant

- Very relevant

5) Long-term negative effects of COVID-19 on the health of the infected is:

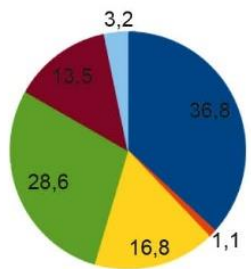

I do not know - I do not have relevant data

- Virtually non-existent

In Comparable to the influenza

- Greater than of the influenza

- Several times greater than of the influenza

= By an order of magnitude greater than of the influenza
2) Face coverings as a blanket measure currently implemented (at the time of answering the survey):

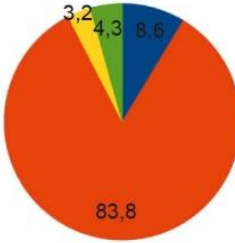

- I do not know - I do not have relevant data

- In total help the population under the current circumstances and decrease

In total are rather detrimental to the population under the current circumstances and increase the
health risks for the individuals

- Do not have any impact

4) The data on the percentage of the Czech population that was already exposed to the virus (regardless the availability of such data) is / would be:

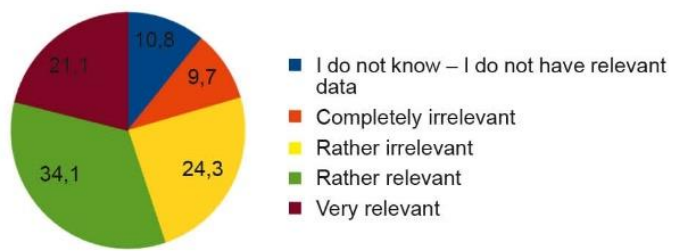

6) By your estimate, what percentage of the Czech population has been already infected with SARS-CoV-2?

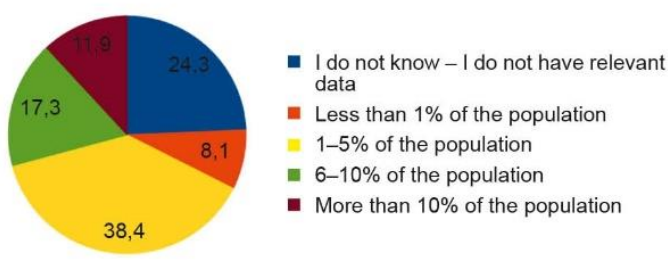

Pic 2 - The results of the second part of the questionnaire rendered as a graph

7) The measures implemented due to the epidemic in the Czech Republic (at the time of answering the survey) are:

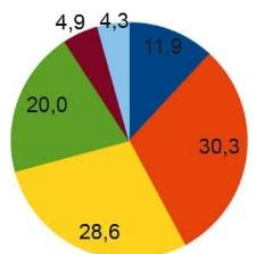

I do not know - I do not have relevant data

- Relevant but insufficient

In Relevant and sufficient

nelevant but excessive

- Irrelevant and excessive

IIrrelevant and absurd
8) According to my opinion, blanket measures implemented as a reaction to the epidemic when in comparison with hypothetical targeted and localised measures brought and will bring to the Czech population:

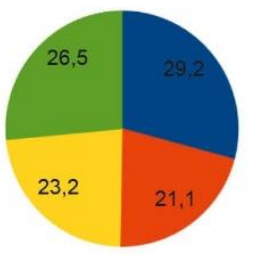

- Ido not know - I do not have relevant data

- More negative health effects than positive

In total approximately the same amount of negative and positive effects

- More positive health effects than negative

Pic 3 - The results of the third part of the questionnaire rendered as a graph 
e) The percentage of the "I do not know - I do not have relevant data" answer in the second part of the questionnaire, where participants were selecting statements that according to them best corresponded to credible data is presented in Tab 2.

Tab 2 - The proportion of the "I do not know - I do not have relevant data" answer in the total of responses for each question.

\begin{tabular}{|l|r|}
\hline $\begin{array}{l}\text { The order of the answer } \\
\text { in the second part of } \\
\text { the questionnaire }\end{array}$ & Percentage \\
\hline 1 & $11.8 \%$ \\
\hline 2 & $8.6 \%$ \\
\hline 3 & $8.1 \%$ \\
\hline 4 & $10.8 \%$ \\
\hline 5 & $37.1 \%$ \\
\hline 6 & $24.2 \%$ \\
\hline
\end{tabular}

f) The differences between the more related and less related fields are presented in Pic 4 . As more related fields are classified $15 \%$ of submitted questionnaires. ${ }^{8}$ These were answered by respondents who stated such fields and specializations that can be regarded as thematically related to the biological and medical aspect of the COVID-19 pandemic. These would include biochemistry, molecular biology, cellular biology, genetics, drug design, pharmacology, molecular toxicology, infectious metallomics, and more. ${ }^{9}$ The other $85 \%$ of respondents claimed specializations that can be regarded as thematically further from the biological and medical aspect of the pandemic, these are designated as less related fields. Among these would be geology, geography, mathematics, optics, botany, ecology, informatics, and more.

\footnotetext{
8 Precisely $14,6 \%$.

${ }^{9}$ Answers included in this category: chemistry - neurochemistry; biochemistry - molecular toxicology and pharmacology; molecular and cellular biology - nuclear receptors, toxicology, pharmacology; physics biophysics; biology - genetics; biology - virology, genetics; biophysics; biochemistry - plant biochemistry; molecular biology; genetics - cytogenetics; physical chemistry - theoretic chemistry and bioinformatics; pharmacy - pharmacology, toxicology; biochemistry - genomics, biochemistry, physical chemistry - drug design; biochemistry - enzymology; biology - toxicology, biology - biology and biotechnology; biochemistry plant and insect biochemistry; informatics - bioinformatics; biophysics - molecular biophysics, pharmacology, molecular and cellular biology; analytic chemistry - infectious metallomics; chemistry - biochemistry; biology - molecular biology. We would like to note that it was impossible to avoid a subjective attitude of the authors. It was also impossible to verify the veracity of respondents' stated fields and specializations.
} 
1) COVID-19 when compared with the seasonal flu is for the population of the Czech Republic:

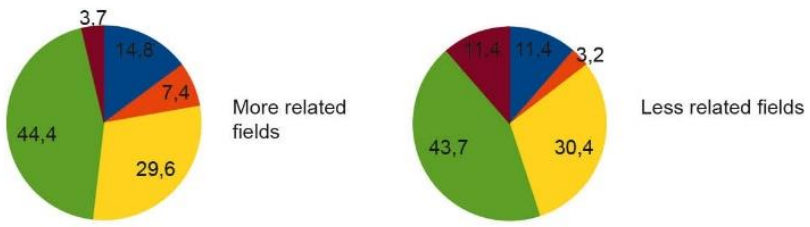

- I do not know - I do not have relevant data

- Less dangerous

Comparably dangerous

- Several times more dangerous

- By an order of magnitude more dangerous

2) Face coverings as a blanket measure currently implemented (at the time of answering the survey):

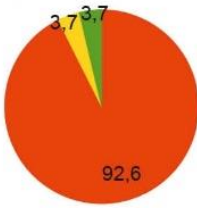

More related fields

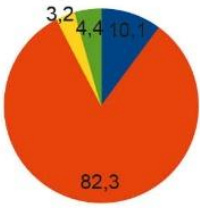

Less related fields

3) Daily updates on the number of detected COVID-19 positive cases are:
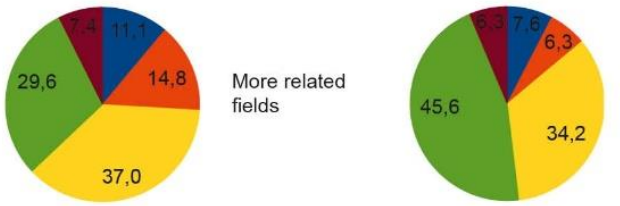

Less related fields

- I do not know - I do not have relevant data

- Completely irrelevan

Rather irrelevant

- Rather relevant

- Very relevant

I do not know - I do not have relevant data In total help the population under the current for the individuals In total are rather detrimental to the population the the risks for the individuals

- Do not have any impact

4) The data on the percentage of the Czech population that was already exposed to the virus (regardless the availability of such data) is / would be:
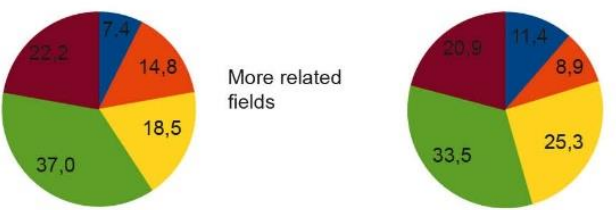

Less related fields

- I do not know - I do not have relevant data

- Completely irrelevant

Rather irrelevant

- Rather relevant

- Very relevan

5) Long-term negative effects of COVID-19 on the health of the infected is:
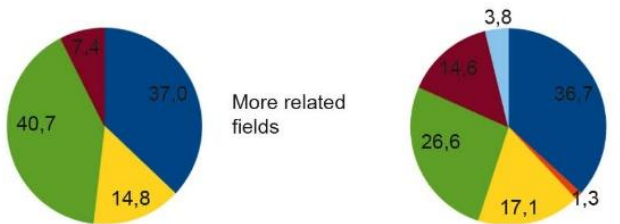

Less related fields

- I do not know - I do not have relevant data

- Virtually non-existent

Comparable to the influenza

- Greater than of the influenza

- Several times greater than of the influenza - By an order of magnitude greater than of the
influenza

6) By your estimate, what percentage of the Czech population has been already infected with SARS-COV-2?
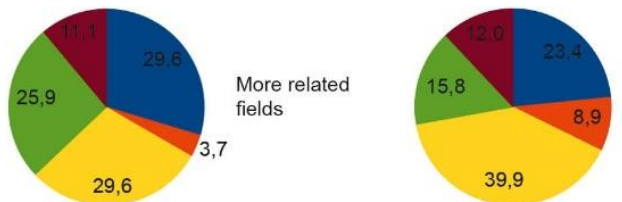

Less related fields

I do not know - I do not have relevant data

- Less than $1 \%$ of the population

(11- $1-5 \%$ of the population

= $6-10 \%$ of the population

- More than $10 \%$ of the population

7) The measures implemented due to the epidemic in the Czech Republic (at the time of answering the survey) are:
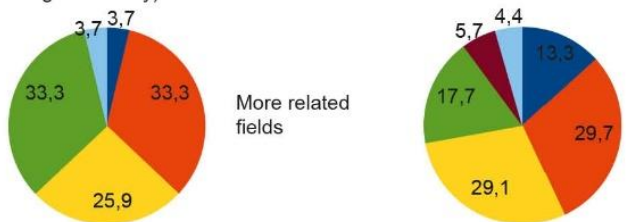

Less related fields

- I do not know - I do not have relevant data

- Relevant but insufficient

Relevant and sufficient

- Relevant but excessive

- Irrelevant and excessive

IIrrelevant and absurd

8) According to my opinion, blanket measures implemented as a reaction to the epidemic when in comparison with hypothetical targeted and localised measures brought and will bring to the Czech population:
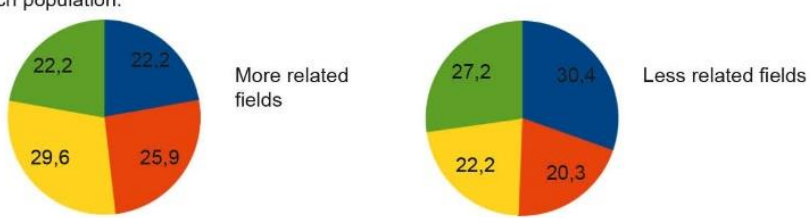

- I do not know - I do not have relevant data

- More negative health effects than positive

In total approximately the same amount of

n More positive health effects than negative

\section{Pic 4 - The ratios between more and less related fields depicted in graphs}


g) The concept of a paradigm is in the study applied in a concrete and specific manner. It is a heuristic attempt at simplifying the discourse into a set of concrete answers through surveying predetermined questions. These are selected in such a way as to interconnect the second and third part of the questionnaire and to amplify the potential variance in the perception of the course of the COVID-19 pandemic in the Czech Republic.

A representative of Paradigm $A$ is a respondent who answered Question 1 (comparison of COVID-19 with influenza) as COVID-19 is for the Czech population less than or comparably dangerous as the seasonal flu, answered that $6 \%$ or more of the population have already been infected and that the government measures implemented in the Czech Republic are either "relevant but excessive", "irrelevant and excessive" or "irrelevant and absurd". ${ }^{10}$

A representative of Paradigm $B$ is a respondent who answered Question 1 (comparison of COVID-19 with influenza) COVID-19 is for the Czech population several times or by an order of magnitude more dangerous than influenza, answered that less than $5 \%$ of the population were already infected and that the government measures implemented in the Czech Republic are either "relevant but insufficient" or "relevant and sufficient". ${ }^{11}$

Answers that fulfil the definition of Paradigm A gave 14 respondents in total, i.e. $7.6 \%$ of participants. Answers that fulfil the definition of Paradigm B gave 44 respondents, i.e. $23.8 \%$ of participants.

h) The answers were processed with a data clustering algorithm using the DENCLUE method $^{12}$ to identify possible clusters of similar answers, i.e. groups of respondents who filled in the questionnaire very similarly. From the data emerged only one significant cluster of similar answers. The cluster was comprised out of 18 respondents (i.e. $9.7 \%$ of the total number of participants). The answers of this group were (when simplified) as follows:

COVID-19 is (Question 1) several times more dangerous than influenza (18x); face coverings (Question 2) in total do help (18x); daily updates on the number of infected (Question 3) are rather relevant (17x) or rather irrelevant (1x); the data showing the exposure rate in the Czech population (Question 4) would be rather relevant (17x) or completely irrelevant (1x); long-term negative health effects of COVID-19 (Question 5) when compared to influenza is greater $(12 x)$ or several times greater $(3 x)$, by an order of magnitude greater $(1 \mathrm{x})$ or comparable $(1 \mathrm{x})$ or I do not know $(1 \mathrm{x})$; the estimate of the rate of exposure in the Czech population (Question 6) is $1-5 \%(12 x)$ or $\mathbf{6 - 1 0 \%}(5 x)$ or more than $10 \%(1 \mathrm{x})$; measures effective in the Czech Republic at the time of submitting the questionnaire (Question 7) are relevant but insufficient (9x) or relevant and sufficient

(6x) or relevant but excessive (3x); blanket measures in comparison with targeted measures bring (Question 8 ) approximately the same amount of negative and positive effects $(11 x)$ or more positive effects $(4 x)$ or I do not know $(2 x)$ or more negative effects $(1 \mathrm{x})$.

\footnotetext{
10 For the exact wording of questions and answers consult Pic 4.

11 For the exact wording of questions and answers consult Pic 4 .

12 Authors utilized the implementation published at 


\section{DISCUSSION}

Utilizing the data from the respondents this study presents a quantification of perceptions of a predefined group of Faculty of Science, Palacký University Olomouc employees towards the evolution of the COVID-19 pandemic in the Czech Republic as representative of the segment of the population that is more experienced at distinguishing the quality and relevancy of data than the general population.

The results indicate that the majority of respondents $(54.1 \%)$ consider the disease to be several times or even by an order of magnitude more dangerous for the Czech population than influenza. On the other hand, there is also a significant group of respondents (34.1\%) who presume that COVID-19 is comparably or even less dangerous. Although it was not the aim of the study to quantify the prevailing media discourse, ${ }^{13}$ it can be said that such a strong representation of the second group is surprising. The high percentage $(83.8 \%)$ of those who consider wearing face coverings inside (a blanket measure in effect at the time of submitting the questionnaire) to be a good policy that helps the population is in accordance with the prevalent media discourse.

Responses to the daily updates of newly infected favoured by the media gave equivocal results. Almost a half of respondents (49.7\%) deem it rather relevant or very relevant, on the other hand $42.2 \%$ consider it rather irrelevant or completely irrelevant. The "rather" relevant variant is prevalent in both opinion groups.

The ratio between the "rather" and "very/ completely" options differs somewhat in Question 4 that asked about the relevancy of exposure data. The "very relevant" option was chosen by 3 times more respondents than the same option in the newly infected question, in total $21.1 \%$. When combined with the "rather relevant" option, these constitute $55.2 \%$ of answers. On the contrary the "rather" and "completely irrelevant" option chose $34.0 \%$ of respondents. From these results we conclude that the focus group is inclined to consider the exposure rate data to be somewhat more significant than the newly infected data. However, this data is not available and as of 10 October 2020 the authors were not aware of any study that would research this. These findings seem substantial in relation to the aim of our study.

The widely discussed topic of the exposure was addressed in Question 6. In this question, respondents were to give their opinion, based on data they view as relevant, on the percentage of the population that has already been infected. Given the unavailability (or a very low number) of direct studies, we expected a high number of respondents choosing the "I do not know - I do not have relevant data"; in reality $24.3 \%$ of respondents chose this option. Nearly half of respondents (46.5\%) assume that at the time of filling in the questionnaire $5 \%$ or less of the Czech population has been already infected. Almost a third of respondents $(29.2 \%)$ believe that $6 \%$ or more has been infected.

As for the quantification of perceptions towards the potential long-term adverse health effects of the COVID-19 disease (Question 5), the dominant answer was as expected "I do not know - I do not have relevant data". This was also the question where the "I do not know - I do not have relevant data" option was selected by the highest number of respondents (36.8\%). The results do not show whether the rest of the respondents believe that there is enough relevant data already or if they wished to share their opinion even without relevant data.

\footnotetext{
${ }^{13}$ Also the influenza illness was not defined in the questionnaire.
} 
The third part of the questionnaire asked the respondents their subjective opinions on government measures. The majority $(78.9 \%)$ chose the options depicting these as relevant for the COVID-19 pandemic. The answers "irrelevant and excessive" and "irrelevant and absurd" chose $9.2 \%$. These results strongly parallel the results in Question 2 asking about face coverings. ${ }^{14}$

The comments from the comment section could be quantified only to a certain degree (as shown in Tab 1). Our assumption that the focus group would comment in strong numbers on the data, their quality or relevancy did not prove correct. The low number of comments did not facilitate quantification. A comment or a link to peer-reviewed or impacted sources shared only two participants (approximately $1 \%$ ).

The ratio of the "I do not know - I do not have relevant data" answers are presented in Tab 2. In general, it can be asserted that respondents largely favoured the other options. It cannot be inferred whether the respondents were truly convinced that they possess a sufficient amount of relevant data for their conclusions. From the subjective comments we conclude that participants decided to share their opinions even when these were not grounded in relevant data, which was against the instructions.

The division into more and less related fields on the basis of the potential affinity to the biological or medical aspects of the pandemic yielded some results. Probably the most important is that with some exceptions we did not observe significant differences between the responses of these two groups. The concrete variances are displayed in Results, here we mention only selected aspects. Question 1 shows that more related fields $(3.7 \%)$ opted for the answer that COVID-19 is by an order of magnitude less dangerous than seasonal flu significantly less than less related fields (11.4\%). The respondents from the more related fields group were also more inclined to consider the daily updates on newly infected (Question 3) as irrelevant, especially in the "completely irrelevant" option (6.3\% less related fields, $14.8 \%$ more related fields). Mild scepticism of the more related fields group toward the character or extent of measures taken by the Czech government could be detected from Question 7, where the sum of answers "relevant but excessive", "irrelevant and excessive" and "irrelevant and absurd" amounted to $37.0 \%$ (27.8\% less related fields). When compared with the less related group, the extra votes are comprised out of votes for the "I do not know" answer and not the other options (Pic 4).

To summarize, in relation to the less related fields the more related fields group in total considers COVID-19 to be dangerous to a lesser degree and the daily infection updates to be somewhat less relevant. Position of this group on blanket government measures is somewhat more critical. These differences do not attest the closeness of these opinions to any reality or truth, they only capture the quantified differences between the positions of academics according to a subjective predetermined formula. The majority of these differences did not reach statistical significance.

The number of respondents fulfilling the criteria of predefined paradigms specified in Results proves solely that such respondents exist. To determine the statistical significance, we compared answers with a statistically random result in case there was no correlation between answers. 14 respondents opted for Paradigm A, while the expected random statistical occurrence would be 5 respondents. 44 respondents opted for Paradigm $B$, while the expected random statistical occurrence would be 27 . We can infer that the

\footnotetext{
${ }^{14}$ Question 8 was not assessed due to a semantic error in its original phrasing.
} 
causations for the respondents to pick their answers to defining questions are not independent of each other. The paradigms, therefore, to a certain degree corelate with opinion groups in the population that manifest themselves by a distinct discourse. Approximately 1 out of every 3 respondents fits with their answers into one of the paradigms. The ration of the paradigms is approximately 1:3 (14 for Paradigm A to 44 for Paradigm B).

An attempt to utilize algorithmic clustering to identify groups of respondents answering the questionnaire offered another approach to defining opinion groups of a certain discourse. This approach would identify opinion groups naturally from results gained from the data and not artificially as is the case with paradigms. This attempt did not result in any outcomes. As is stated in chapter Results, only one cluster of answers was identified and this cluster had very differing answers to Questions 7 and 8, that is subjective question in the third part of the questionnaire. 


\section{Conclusion}

The study presents a heuristic quantification of perceptions of the respondents towards the evolution of the COVID-19 pandemic in the Czech Republic and introduces certain devices that can be used to analyse it. Some final data can be correlated with the media discourse (or discourses) or public paradigms.

Certain usefulness of the survey on the topic of the health care aspect of the current situation is indicated by the complexity of the issues related to the COVID-19 pandemic in the Czech Republic, and also by the reality of the post-factual age when a significant space is given to misleading, confusing, in some cases positively false news. The complexity of the issue cannot be descried by members of the respective specializations, no matter if these are epidemiologists, virologists or biochemists on one hand, or economists, sociologists or psychologists on the other hand. We should not presume either that the issue can be fully and correctly understood by groups comprised of multiple specialists advising members of the government. Peer-reviewed data surely provide a strong basis for a number of qualified opinions, but we see instances where different specialists interpret the same data in a different manner and ultimately draw from them even diametrically different conclusions. The metanarrative approach one could wish to employ in the data processing is an illusion.

Due to the above stated, the decisions in the real socioeconomical and health care areas cannot be anything but political. Interviewing other groups of more or less informed academics from various fields and with various experience in reviewing and evaluating data can be utilised as an instrument to eliminate with a degree of probability entirely erroneous opinions, invalid procedures or propositions of solutions sponsored by groups whose motivation might be covert and could be nonbeneficial to the population as a whole. Survey results cannot be, for obvious reasons, an argument for any decision or measure, but they can cocreate a rudimentary level of certainty in the process of potential correction for seemingly predominant public opinion paradigms, which dominance can manifest itself in the media sphere, or in the process of their confirmation. Strategy and policy makers are under a significant and possibly contradicting pressure from different interest groups and the general public. Surveys among academics and specialists offer a way to offset these with a more balanced and fact-based outlook on difficult and complex issues.

This heuristically performed suggestion of a survey of perceptions of FoS UPOL academics indicates that the potential of such surveys to correct for the prevailing public and media paradigm is not null. It would be necessary to design the questionnaire in cooperation with sociologists and members of the field related to the topic of the survey. Results valuable to policy and strategy makers and health care management could be gained if the respondents were for example physicians and researchers from faculties of medicine, or medical practitioners from the Czech Medical Chamber. To assess the media influence, it could be valuable to compare the opinion and position differences between the general population and natural scientists, medical practitioners or other groups. 